\section{Parto vertical em hospital universitário: série histórica, 1996 a 2005}

\section{Vertical-position births at a University Hospital: a time-series study, 1996 to 2005}

\section{Abstract}

Objectives: to describe the evolution of the number of horizontal and vertical births in the maternity ward of the University Hospital of the Federal University of Santa Catarina, Brazil and to evaluate their correlation with the rates for caesarian, for transfer of newborns to intensive and semi-intensive care units, and maternal blood transfusions.

Methods: a time-series study. All births resulting in newborns being transferred to the Intensive Care Unit, and the maternal blood transfusions obstetrics ward between 1996 and 2005 were included in this study. In order to test the tendencies, the PraisWinsten generalized linear regression method was used.

Results: in 1996 the percentage for vertical births was $5.4 \%$ and in $200552.3 \%$. The average annual variance for vertical births was $+20.8 \%(p=0.007)$, and for horizontal births $-15.2 \% \quad(p<0.001)$. Caesarian births showed a tendency to stabilize $(p=0.243)$. There was a decrease of in the number of newborns transferred to the neonatal intensive care unit, $6.1 \%$ per year $(p=0.001)$ and in the need of maternal blood transfusions $(5.2 \%-p<0.01)$.

Conclusions: the growing percentage of vertical births is in accordance with scientific evidence and the World Health Organization's recommendations. This practice has been gradually introduced by the team of health care professionals.

Key words Labor stage, second, Natural childbirth, Time series studies, Humanizing delivery
Odaléa Maria Brüggemann 1

Roxana Knobel 2

Eli Rodrigues Camargo Siebert 3

Antônio Fernando Boing 4

Halana Faria de Aguiar Andrezzo 5

1 Programa de Pós-Graduação em Enfermagem. Departamento de Enfermagem. Universidade Federal de Santa Catarina. Centro de Ciências da Saúde. Campus Universitário. Trindade. Florianópolis, SC, Brasil. CEP: 88.040-970 E-mail: odalea@nfr.ufsc.br 2 Departamento de Tocoginecologia. Universidade Federal de Santa Catarina. Florianópolis, SC, Brasil

3 Serviço de Enfermagem. Centro Obstétrico da Maternidade do Hospital Universitário. Universidade Federal de Santa Catarina. Florianópolis, SC, Brasil

4 Departamento de Saúde Pública. Universidade Federal de Santa Catarina. Florianópolis, SC, Brasil

5 Programa de Saúde da Família. Prefeitura Municipal de Biguaçu. Florianópolis, SC, Brasil.

\section{Resumo}

Objetivos: descrever a evolução do número de partos horizontais e verticais na maternidade do Hospital da Universidade Federal de Santa Catarina, Brasil, e avaliar a associação dos mesmos com a taxa de cesárea, de internações dos recém-nascidos em unidade de tratamento intensivo e semi-intensivo e as transfusões sanguineas maternas.

Métodos: estudo descritivo - série histórica. Foram incluidos todos os partos, as internações dos recémnascidos na Unidade de Terapia Intensiva e as transfusões sanguineas maternas ocorridas de 1996 até 2005. Para testar as tendências, utilizou-se o método de PraisWinsten para regressão linear generalizada.

Resultados: em 1996 a porcentagem de partos verticais era 5,4\% e em 2005 foi 52,3\%. A variação média anual dos partos verticais foi de $+20,8 \%(p=0,007)$ e dos partos horizontais de $-15,2 \%(p<0,001)$. Os partos cesáreos apresentaram tendência de estabilidade. Houve diminuição no número de recém-nascidos internados na unidade de terapia intensiva neonatal de $6,1 \%$ ao ano ( $p=0,001)$ e de transfusões sanguineas ou hemoderivados $(5,2 \%-p<0,01)$.

Conclusões: o aumento de partos verticais em relação aos demais está em consonância com as evidências científicas e recomendações da Organização Mundial da Saúde. Essa prática foi incorporada gradativamente pelos profissionais da equipe de saúde.

Palavras-chave Segunda fase do trabalho de parto, Parto Normal, Estudos de séries temporais, Parto humanizado 


\section{Introdução}

Conforme o momento histórico e tipo de assistência obstétrica prestada, observam-se diferenças marcantes na posição materna considerada mais adequada para o período expulsivo. Parece que, tanto na antiguidade da maioria das civilizações, quanto em culturas primitivas, a posição materna preferencial era a vertical. ${ }^{1}$

Com a medicalização do parto, a posição deitada e com as pernas colocadas em perneiras (litotomia) passou a ser considerada mais adequada, pelas restrições impostas pelos próprios procedimentos hospitalares, como a monitorização fetal, a terapia endovenosa e a analgesia, entre outros. Também por facilitar o controle da progressão fetal e a realização de um parto operatório, por parte do profissional, em caso de necessidade. 1

O uso da posição horizontal materna no momento do período expulsivo, a exemplo do que ocorreu com outras intervenções obstétricas, foi adotado de maneira indiscriminada, sem a devida avaliação de sua efetividade ou segurança.2,3

A partir do final do século 20, houve em todo o mundo um movimento para oferecer uma assistência à saúde baseada na evidência da segurança e da efetividade dos procedimentos. ${ }^{2}$ Este processo tem como marco conceitual a chamada "medicina baseada em evidências", que envolve o uso de metaanálises e revisões sistemáticas, preferencialmente derivadas de estudos randomizados controlados, como instrumentos de sumarização sistemática das pesquisas existentes. 4,5

A avaliação das práticas obstétricas e recomendações relacionadas à assistência ao parto fundamentadas nas melhores evidências disponíveis foram publicadas, a partir de 1985, pela Organização Mundial da Saúde (OMS). 6 No Brasil, essas recomendações foram amplamente divulgadas com a distribuição realizada pelo Ministério da Saúde dos livros "Assistência ao Parto Normal: um guia prático"6 e "Parto, aborto e puerpério. Assistência humanizada à mulher".7

Ao considerar a melhor evidência científica disponível, a posição vertical é vantajosa, reduzindo a duração do período expulsivo e a queixa de dor severa durante o mesmo, o número de partos operatórios, a necessidade de episiotomia e o número de alterações no batimento cardíaco fetal. ${ }^{1} \mathrm{O}$ uso dessa posição no período expulsivo também está relacionado com um aumento do número de lesões perineais de segundo grau e de perda sanguínea maior que $500 \mathrm{~mL} .1$
O uso rotineiro da posição horizontal durante o período expulsivo foi classificado como uma das práticas claramente prejudiciais ou ineficazes e que deveriam ser eliminadas, ${ }^{6}$ havendo a recomendação praticamente consensual entre os autores de evitá1a. $1,2,7-9$

Com base nas recomendações da OMS para a assistência ao parto, o Ministério da Saude sugere que a mulher deve ser encorajada a adotar a posição que preferir para seu parto, cabendo aos profissionais respeitá-la e possuírem treinamento para utilizá-la. 7

Ressalta-se que a morbidade materna está intimamente relacionada com a mortalidade materna e a antecede; portanto, intervenções capazes de reduzir a morbidade devem também reduzir a mortalidade materna. Dessa forma, a adoção das recomendações baseadas em evidências científicas (entre elas a adoção da posição verticalizada no nascimento) busca não apenas maior conforto e uma experiência de parto mais satisfatória para a mulher, mas também a redução da morbi-mortalidade materna. 5

No Brasil, estão sendo realizadas pesquisas sobre a expectativa e/ou preferência das mulheres sobre a via de parto (vaginal ou cesárea).10,11 Entretanto, a posição de parto em que as mulheres estão sendo assistidas tem sido pouco pesquisada, uma vez que as maternidades, de maneira geral, ainda preconizam a utilização da posição horizontal durante o parto.12,13 Apenas algumas instituições passaram a assistir ao parto na posição vertical e/ou lateral. 14,15

Este estudo tem como objetivo descrever a evolução do número de partos horizontais e verticais na maternidade do Hospital Universitário da Universidade Federal de Santa Catarina (HU/UFSC) e avaliar a associação dos mesmos com a taxa de cesárea, de internações dos recém-nascidos em unidade de tratamento intensivo e semi-intensivo e a necessidade de transfusões sanguíneas maternas.

\section{Métodos}

Trata-se de um estudo descritivo - série histórica. Os dados foram coletados no HU/UFSC, classificado como de médio porte, que presta atendimento de média e alta complexidade, atendendo exclusivamente pelo Sistema Único de Saúde. A maternidade do referido hospital, desde a sua ativação, em outubro de 1995, presta assistência com base em sua filosofia, que tem como princípios a humanização e interdisciplinaridade, sempre vinculadas às práticas recomendadas pela OMS e da melhor evidência disponível. 14 
A assistência ao parto na maternidade do HU/UFSC consiste em propiciar à parturiente a adoção da posição verticalizada no momento do período expulsivo: de cócoras, sentada ou semisentada sem o uso de perneiras. A condução obstétrica no período expulsivo é realizada de acordo com a preconizada para a assistência ao parto normal, o que difere é que nessa posição de parto, as intervenções como a episiotomia e as manobras no desprendimento da cabeça e das escápulas fetais não são realizadas de forma rotineira, e geralmente não são necessárias.

As duas salas para parto normal da maternidade estimulam esse posicionamento, pois possuem cadeiras para parto (que podem também ficar em posição ginecológica tradicional, se necessário). A incorporação dessa prática, pelos profissionais da equipe de saúde, ocorreu de forma gradativa ao vivenciarem e reconhecerem as suas vantagens. A posição de parto adotada sempre respeita a escolha da mulher, podendo ser, inclusive, na posição horizontal ou outra.

Nesse estudo, foram incluídos todos os partos vaginais e cesáreos, o número de internações dos recém-nascidos na Unidade de Terapia Intensiva Neonatal (UTIN) e o número de transfusões de sangue e/ou hemoderivados para pacientes obstétricas (internadas no centro obstétrico ou alojamento conjunto) ocorridos desde o primeiro ano completo de funcionamento, em 1996, até o ano de 2005.

Os dados sobre os partos foram obtidos através da estatística anual de procedimentos do Serviço de Enfermagem do Centro Obstétrico. Já o número de internações de recém-nascidos na UTIN foram obtidos a partir da estatística anual da Seção de Arquivo e Estatística do Serviço de Prontuário do Paciente do HU/UFSC, e a quantidade de transfusões sanguíneas da estatística do banco de sangue. Os dados são expressos em porcentagem com relação ao total de partos (para tipo de parto e número de transfusões) e de nascimentos (para internações na UTIN).

Para testar se as tendências de partos verticais, horizontais e cesáreas, das internações de recémnascidos e das transfusões sanguíneas para pacientes obstétricas foram declinantes, ascendentes ou estacionárias entre 1996 e 2005, utilizou-se o método de Prais-Winsten para regressão linear generalizada. Este teste corrige o efeito de autocorrelação de primeira ordem e quantifica a variação anual das proporções. Considerou-se estacionária a tendência cujo coeficiente de regressão não foi diferente de zero $(p>0,05)$ e foram também calculados os inter- valos de confiança (95\%). O pacote estatístico Stata 9 foi utilizado nas análises.

O protocolo de pesquisa foi aprovado pelo Comitê de Ética em Pesquisa da UFSC, parecer no 186/06 e autorizado pela diretoria da instituição. Todos os aspectos que envolvem a pesquisa estão de acordo com a Resolução no 196/96 do Conselho Nacional de Saúde.

\section{Resultados}

No período do estudo ocorreram 15.891 partos, sendo 4998 cesáreos e 10.893 partos vaginais (5549 assistidos em posição vertical e 5344 horizontal) de acordo com a Tabela 1. Houve significativa variação na posição em que as parturientes foram assistidas na segunda fase do parto normal, com aumento na proporção de partos verticais e um declínio de partos horizontais (Figura 1). A menor porcentagem de partos verticais em relação aos demais tipos foi no ano de 1996 (83 - 5,5\%) e a maior em 2005 (764 $52,3 \%$ ). Houve uma relação inversa no que se refere ao parto horizontal, ou seja, a maior porcentagem foi em 1996 (961 - 62,9\%) e a menor em 2005 (202 $13,8 \%$ ) (Tabela 1). Nestes dez anos estudados a variação média anual dos partos verticais foi de $+20,8 \%$ $(p=0,007)$ e dos partos horizontais de $-15,2 \%$ $(p<0,001)$ (Tabela 2).

Já os partos cesáreos apresentaram tendência de estabilidade $(p=0,243)$. A menor porcentagem de cesáreas ocorreu em 2001 (480 - 28,2\%) e a maior em 2004 (561 - 37,4\%), como se pode obeservar nas Tabela 1 e 2 . Até 2002 a porcentagem de cesarianas permaneceu inferior a $32 \%$, no entanto, no ano de 2003 houve uma elevação e os valores não retornaram mais aos identificados para os anos iniciais do estudo (Tabela 1, Figura1).

Houve uma contínua ascensão da porcentagem de partos verticais até o ano de 2002, com uma queda em 2003 e 2004 e posterior ascensão. Com relação ao parto horizontal, ocorreu um declínio na porcentagem no período estudado, com uma discreta elevação no ano de 2003 (Tabela 1, Figura 1).

$\mathrm{O}$ número de internações dos recém-nascidos na Unidade de Terapia Intensiva Neonatal (UTIN) foi de 2771. Em 1996, aproximadamente 31\% (502) dos recém-nascidos foram internados na UTIN. Já no ano seguinte (1997) o número reduziu aproximadamente pela metade (268). Com exceção do ano de 1998 (318 internações), no qual houve um pequeno aumento, o número de recém-nascido internados na UTIN manteve-se estável em torno de $15 \%$, o que representou uma média de diminuição de $6,1 \%$ ao ano, o que foi estatisticamente significante $(p=0,01)$ (Tabela 1 e 2). 


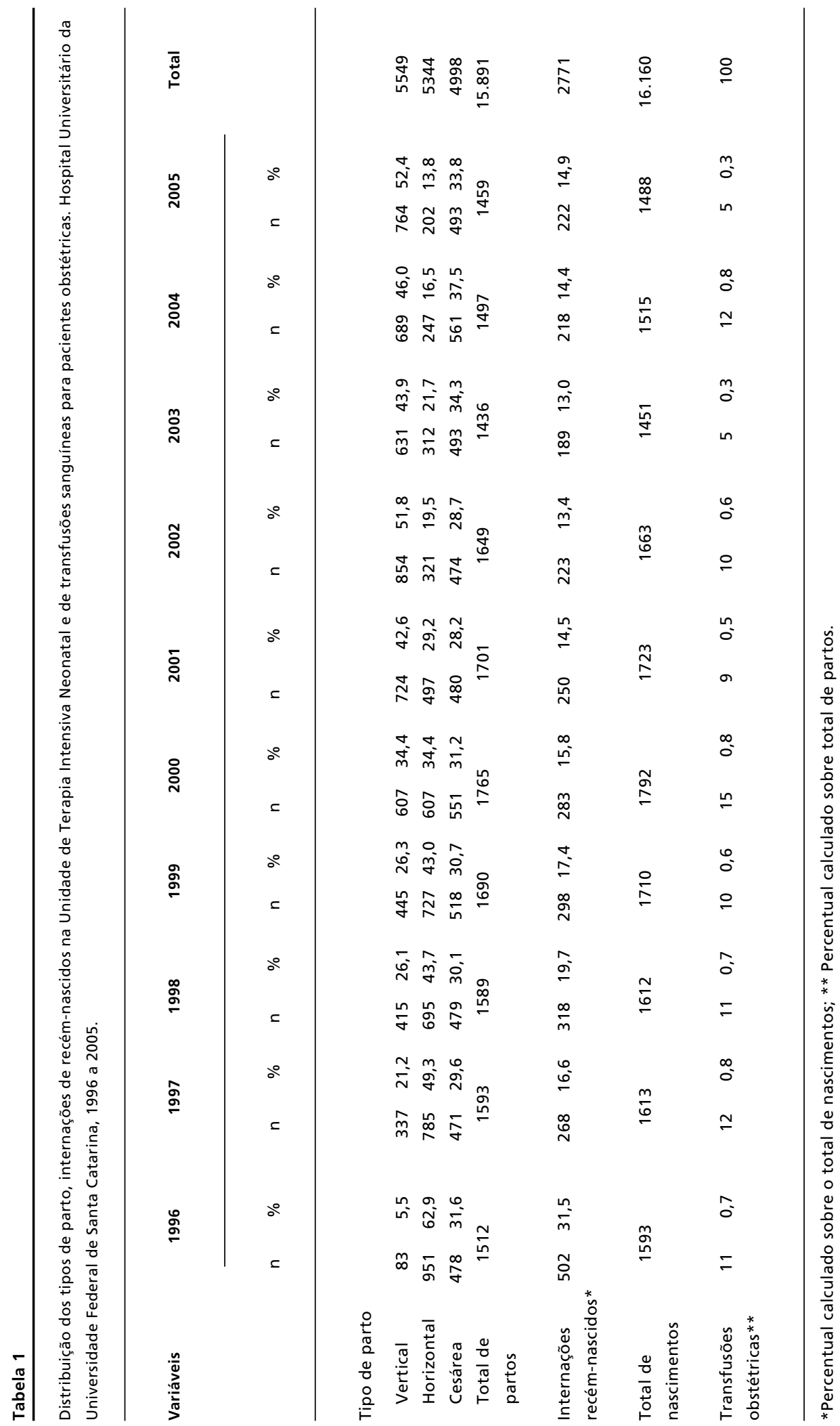


Série histórica dos partos verticais, horizontais e cesáreas. Hospital Universitário da Universidade Federal de Santa Catarina, 1996 a 2005.

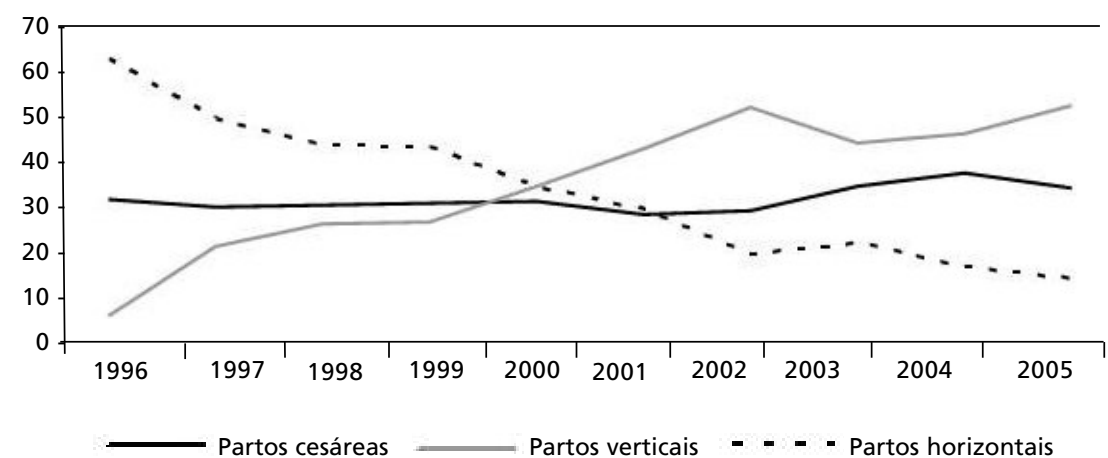

Tabela 2

Tendência dos partos, da internação de recém-nascidos na Unidade de Terapia Intensiva Neonatal e de transfusões sanguíneas para pacientes obstétricas. Hospital Universitário da Universidade Federal de Santa Catarina, 1996 a 2005.

\begin{tabular}{|c|c|c|c|c|c|}
\hline & \multirow[t]{2}{*}{ Variação anual \% } & \multicolumn{2}{|c|}{$\mathrm{IC95 \%}$} & \multirow[t]{2}{*}{$p^{*}$} & \multirow[t]{2}{*}{ Interpretação } \\
\hline & & Inferior & Superior & & \\
\hline \multicolumn{6}{|l|}{ Tipo de parto } \\
\hline Vertical & $+20,8$ & $+10,3$ & $+32,3$ & 0,007 & Acréscimo \\
\hline Horizontal & $-15,2$ & $-16,5$ & $-13,8$ & $<0,001$ & Decréscimo \\
\hline Cesárea & $+1,4$ & $-0,5$ & $+3,4$ & 0,243 & Estabilidade \\
\hline Internações recém-nascidos & $-6,1$ & $-9,1$ & $-2,9$ & 0,01 & Decréscimo \\
\hline Transfusões obstétricas & $-5,2$ & $-3,3$ & $-7,2$ & $<0,01$ & Decréscimo \\
\hline
\end{tabular}

* Teste de Prais-Winsten.

Foram realizadas 100 transfusões de sangue e/ou hemoderivados para pacientes obstétricas no período do estudo. Embora com grandes variações, esse número também apresentou uma diminuição de $5,2 \%$ ao ano, e esta diferença foi estatisticamente significante $(p<0,01)$ (Tabela 1 e 2$)$.

\section{Discussão}

A crescente porcentagem de partos verticais em relação aos demais tipos de parto encontrados na presente série histórica estão em consonância com as recomendações da OMS e Ministério da Saúde,2,6,7 ou seja, que a mulher deve ser estimulada a experimentar a posição desejada por ela durante o segundo estágio do trabalho de parto, evitando longos períodos em decúbito dorsal, e sua escolha deve ser apoiada.

Faltam dados publicados para comparar os resultados obtidos neste estudo com outros hospitais e até mesmo com outras populações. $\mathrm{Na}$ literatura nacional, as pesquisas sobre taxas de parto têm tido como foco a via de parto (normal ou cesariana). São escassos os dados sobre essa prática, entretanto, pelo senso comum, tem-se conhecimento de algumas instituições ou grupos que buscam incorporar as evidências científicas sobre a assistência ao parto e adotam em suas condutas a posição vertical ou lateral para o parto. Os dados publicados se limitam às experiências de centro de parto normal intrahospitalar, no qual as posições não supinas (lateral e vertical) foram adotadas em $55 \%$ dos partos 
normais, 15 e de casa de parto, na qual os partos na posição semi-sentada, sentada, de cócoras e vertical corresponderam a $47,75 \%$ do total de partos no período estudado. ${ }^{16}$ Ambas as experiências, embora em outros cenários de atenção obstétrica, se assemelham aos achados do presente estudo.

Mesmo na literatura internacional, são poucas as informações encontradas. Dados de uma pesquisa americana envolvendo 1583 mulheres, das quais $74 \%$ tiveram parto vaginal, mostram que $23 \%$ delas foram assistidas na posição vertical. ${ }^{17}$

Um estudo sobre o tema em hospitais públicos da Tanzânia concluiu que a posição supina é a mais utilizada para o parto em todos os hospitais pesquisados, mas que essa é a escolha da maioria das parturientes. 18 Dados semelhantes foram obtidos em partos hospitalares assistidos na China. 19

O número de cesáreas permaneceu estável em torno de $30 \%$ até 2002 , quando houve um pequeno aumento. Essa frequência de cesárea, bem acima da recomendada pela OMS, segue uma tendência mundial que também é observada em hospitais públicos no Brasil, no mesmo período de tempo.10,11 Diversos podem ser os fatores associados com essas taxas de cesáreas elevadas, entre eles o fato de ser um hospital terciário, com uma maior proporção de gestantes com complicações clínicas e/ou obstétricas, especialmente oriundas dos municípios do interior do estado de Santa Catarina. Fatores relacionados com o ensino médico, com o saber e a forma como a obstetrícia tem sido praticada, além da crença de que cesárea é um parto "mais seguro", resolutivo e mais desejado pelas mulheres podem estar influenciando a decisão da via de parto. Pelo fato de o hospital buscar uma assistência com base nas evidências científicas, sugerem-se outras investigações que possam avaliar as causas dessa elevada taxa.

A posição vertical está relacionada ao aumento da perda sanguínea materna, como apontam as evidências científicas. ${ }^{1}$ Embora não tenha sido avaliada a perda sanguínea das parturientes nesse estudo, a necessidade de transfusão sanguínea e de hemoderivados para as mulheres internadas no centro obstétrico e alojamento conjunto apresentou uma tendência à diminuição durante os dez anos estudados. Esse fato pode estar relacionado com outras variáveis não relacionadas ao estudo ou a subregistro dos dados, mas de forma indireta demonstra que o aumento dos partos assistidos em posição vertical não levou ao aumento da necessidade de sangue ou hemoderivados.

A taxa de internação de recém-nascidos em UTIN inicialmente diminuiu (1997) e depois permaneceu estável. Esses dados não permitem avaliar a causa da internação individualmente, se houve relação com a posição assumida pela mulher no parto ou se houve uma diminuição da porcentagem de internações por outros motivos. Na prática obstétrica, observa-se uma preocupação quanto à segurança para o feto da assistência ao parto em outras posições que não a tradicional, uma vez que o decúbito dorsal favorece a instrumentação do parto, quando necessária, e avaliação fetal.1,20 Acredita-se que, se posição vertical de parto interferisse na vitalidade do produto conceptual, o número de internações teria aumentado proporcionalmente ao número de partos não horizontais, o que não ocorreu nesse estudo. Apesar das limitações do método de pesquisa, os dados obtidos são congruentes com os encontrados em ensaios clínicos e revisões sistemáticas, que enfatizam a segurança da posição vertical no segundo período para o feto/recémnascido. $1,8,21$

Parece ainda existir uma incongruência entre o que recomendam as evidências científicas e a forma como as práticas de assistência ao parto são planejadas e desenvolvidas, embora se reconheça a importância dos ensaios clínicos e revisões sistemáticas sobre as mesmas. Os estudos que avaliam a implementação das recomendações da OMS (entre elas a adoção da posição de escolha da mãe no momento do nascimento) mostram que as mesmas não são adotadas como rotinas na maioria dos hospitais, tanto no Brasil,12,13 como em outros países. 22-24

Há um consenso de que os profissionais que assistem ao parto necessitam de treinamento para atender a parturiente em outras posições, além da supina, para não inibirem a escolha da mulher. ${ }^{2,6}$ Mas, pelo observado em diversos estudos, existe uma dificuldade em sensibilizar profissionais e gestores a adotar medidas que permitam essa prática na assistência hospitalar. 12,13,22-25

Os resultados obtidos no HU/UFSC, mostrados nesse estudo, refletem o exercício prático dos princípios básicos da filosofia que norteiam a assistência prestada na referida instituição, entre os quais destacam-se que:

\footnotetext{
[...] a equipe interdisciplinar que presta assistência à mulher - recém-nascido - família, deve atuar de forma integrada, visando um atendimento adequado...(Santos; 2001: 76). ${ }^{14}$

[...] todo pessoal deve ter qualificação, treinamento e supervisão continuadas, específicas, para prestação da assistência a que tem direito a mãe, o recém-nascido e a família. (Santos; 2001: 76). 14
} 
O processo de acompanhar o parto na posição vertical se iniciou na implantação do serviço, quando a comissão responsável discutia as rotinas hospitalares a serem seguidas; inicialmente, apenas dois obstetras assistiam os partos em posição vertical. Esses obstetras passaram a estimular e apoiar os colegas e as enfermeiras obstétricas a adotarem essa prática, que foi incorporada de forma gradativa por todos os profissionais da equipe de saúde do centro obstétrico, tornando possível respeitar a escolha da mulher em qualquer plantão.

Desde 1996 foi implementada uma rotina de enfermagem que consiste em levar a parturiente e acompanhante para conhecer as salas de parto e orientar sobre as vantagens e desvantagens da posição vertical e horizontal com inclinação. A partir disso, a sua opção é registrada no prontuário, sendo observada e respeitada pela equipe no momento do período expulsivo. Considera-se que a mulher foi protagonista nesse processo de mudança, e que o respeito à sua escolha pela posição vertical contribuiu para os resultados encontrados nessa pesquisa. Assim, colocam-se em prática os princípios de interdisciplinaridade e humanização do atendimento, que norteiam a filosofia da maternidade. Embora não tenha sido objeto desse estudo, entre as vantagens das posições não supinas estão a diminuição da sensação de desconforto no período expulsivo, 1 que pode influenciar na adoção dessa posição pela mulher.

A formação médica tradicional tende a considerar o parto horizontal mais simples e confortável para o obstetra, 1,26 o que parece dificultar a adoção dessa prática em outras maternidades. Por tratar-se de um hospital de ensino, no qual são desenvolvidas as atividades teórico-práticas dos cursos de graduação em enfermagem e medicina, além da residência médica em tocoginecologia, a maternidade do

\section{Referências}

1. Gupta JK, Hofmeyr GJ. Position for women during second stage of labour for women without epidural anaesthesia. Cochrane database of systematic reviews (online) 2004(1): CD002006.

2. Enkin M, Deirse M, Neilson J, Crowther C, Duley L, Hodnett E, Hoffmeyer J. Guia para atenção efetiva na gravidez e no parto. 3. ed. Rio de Janeiro: Guanabara Koogan; 2005.

3. Appropriate technology for birth. Lancet. 1985; 2: 436-7.

4. Haines A, Kuruvilla S, Borchert M. Bridging the implementation gap between knowledge and action for health. Bull World Health Organ [serial online]. 2004; 82: 72432. [consulted on: 20, dec 2007]. Available from: http://www.who.int/bulletin/volumes/82/10/724.pdf
HU/UFSC está contribuindo para a formação de profissionais em consonância com as evidências científicas.

Esse estudo mostra a possibilidade de implementação da assistência ao parto na posição vertical respeitando a escolha da mulher, sem mudanças significativas em relação às internações dos recémnascidos em UTIN e ao número de transfusões maternas.

A partir desses achados, demonstra-se que a posição vertical é uma prática que pode ser considerada segura, uma vez que não acarretou em riscos maternos e fetais, podendo ser incorporada por outras instituições que prestam assistência ao parto. Embora não tenha sido foco desse estudo, ressaltamse as diversas vantagens dessa posição demonstradas por estudo quantitativos analíticos:1,2,8 a redução do tempo, da dor e do desconforto no período expulsivo, além da diminuição de intervenções obstétricas e de padrões anormais nos batimentos cardíacos fetais.

Todas as vantagens descritas colocam a mulher como protagonista do evento do parto, podendo influenciar positivamente sua percepção sobre a experiência que, resguardado o bem- estar materno e fetal, deve ser mais valorizada do que o suposto incômodo referido por alguns profissionais que resistem a assistir o parto na posição vertical. 26

Sugere-se a realização de novas pesquisas aprofundando o tema para avaliar outras variáveis de morbi-mortalidade materna e para o feto/recémnascido que possam estar relacionadas com a posição materna no momento do parto. Mudanças assistenciais são difíceis e desafiadoras, mas são possíveis quando a equipe de profissionais se dispõe a rever condutas habituais, incorporando novas práticas fundamentadas em evidências científicas.
5. Cecatti JG, Calderón IMP. Intervenções benéficas durante o parto para a prevenção da mortalidade materna. Rev Bras Ginecol Obstet. 2005; 27: 357-65.

6. OMS (Organização Mundial da Saúde). Assistência ao parto normal: um guia prático. Genebra; 1996.

7. Brasil. Ministério da Saúde. Secretaria de Políticas de Saúde. Área Técnica de Saúde da Mulher. Parto, aborto e puerpério: assistência humanizada à mulher. Brasília, DF; 2003.

8. De Jonge A, Teunissen TA, Lagro-Janssen AL. Supine position compared to other positions during the second stage of labor: a meta-analytic review. J Psychosom Obstet Gynaecol. 2004; 25: 35-45. 
9. Lothian JA. The coalition for improving maternity services: evidence basis for the ten steps of mother-friendly care. Introduction - The coalition for improving maternity services. J Perinat Educ. 2007; 16 (Suppl): S1-4.

10. Faundes A, Padua KS, Osis MJ, Cecatti JG, Sousa MH. Brazilian women and physicians' viewpoints on their preferred route of delivery. Rev Saúde Pública. 2004; 38 : 488-94.

11. Tedesco RP, Maia Filho NL, Mathias L, Benez AL, Castro VCL, Bourroul GM, Reis FI. Primigravid expectations about the delivery method and the causal factors for their choice. Rev Bras Ginecol Obstet. 2004; 26: 791-8.

12. d'Orsi E, Chor D, Giffin K, Angulo-Tuesta A, Barbosa GP, Gama AS, Reis AC, Hartz Z. Quality of birth care in maternity hospitals of Rio de Janeiro, Brazil. Rev Saúde Pública. 2005; 39: 645-54.

13. Boaretto, MC. Avaliação da política de humanização ao parto e nascimento no município do Rio de Janeiro [dissertação]. Rio de Janeiro: Escola Nacional de Saúde Pública da Fundação Oswaldo Cruz; 2003. [acesso em: 10 jul. 2005]. Disponível em: http://teses.cict.fiocruz.br/cgibin/wxis1660.exe/lildbi/iah/

14. Santos OMB, Siebert ERC. The humanization of birth experience at the University of Santa Catarina maternity hospital. Int J Gynaecol Obstet. 2001; 75 (Suppl 1): S73S9.

15. Schneck CA, Riesco MLG. Intervenções no parto de mulheres atendidas em um centro de parto normal intrahospitalar. Revista Mineira de Enfermagem 2006; 10: 240-

16. Fernandes BM. A casa de parto da Faculdade de Enfermagem de Juiz de Fora: diagnóstico do perfil da assistência e a percepção das usuárias [doutorado]. Rio de Janeiro: Programa de Pós-Graduação da Escola de Enfermagem Anna Nery da Universidade Federal do Rio de Janeiro; 2004.

17. Declercq ER, Sakala C, Corry MP, Applebaum S, Rischer P. Listening to mothers: report of the first nacional US survey of women's childbearing experiences. New York: Maternity Center Association; 2002.

Recebido em 6 de março de 2008

Versão final apresentada em 29 de setembro de 2008

Aprovado em 13 de novembro de 2008
18. Lugina H, Mlay R, Smith H. Mobility and maternal position during childbirth in Tanzania: an exploratory study at four government hospitals. BMC Pregnancy Childbirth. 2004; $4: 3$

19. Qian X, Smith H, Zhou L, Liang J, Garner P. Evidencebased obstetrics in four hospitals in China: an observational study to explore clinical practice, women's preferences and provider's views. BMC Pregnancy Childbirth. 2001; 1: 1.

20. Martins-Costa SH, Vidal AMC, Chaves MTP. Assistência ao trabalho de parto. In: Freitas F, Martins-Costa SH, Ramos JGL, Magalhães JA. Rotinas em Obstetrícia. 5. ed. Porto Alegre: Artmed; 2006. p. 231-46.

21. Terry RR, Westcott J, O'Shea L, Kelly F. Postpartum outcomes insupine delivery by physicians vs nonsupine delivery by midwives. J Am Osteopath Assoc. 2006; 106 : 199-202.

22. Colomar M, Belizan M, Cafferata ML, Labandera A, Tomasso G, Althabe F, Belizán JM, Grupo de Estudio de Prácticas Perinatales. Practices of maternal and perinatal care performed in public hospitals of Uruguay. Ginecol Obstet Mex. 2004; 72: 455-65.

23. Khalil K, Elnoury A, Cherine M, Sholkamy H, Hassanein N, Mohsen L, Miraf Breebaart M, Shoubary AA. Hospital practice versus evidence-based obstetrics: categorizing practices for normal birth in an Egyptian teaching hospital. Birth. 2005; 32: 283-90.

24. Turan JM, Bulut A, Nalbant H, Ortayli N, Erbaydar T. Challenges for the adoption of evidence-based maternity care in Turkey. Soc Sci Med. 2006; 62: 2196-204.

25. Angulo-Tuesta A, Giffin K, Gama Ade S, d'Orsi E, Barbosa GP. Cooperation and conflict in childbirth care: representations and practices of nurses and obstetricians. Cad Saúde Pública. 2003; 19: 1425-36.

26. Teixeira NZF, Pereira WR. Parto hospitalar: experiência de mulheres da periferia de Cuiabá-MT. Rev Bras Enferm. 2006; 59: 740-4. 